\title{
Respuestas de los murciélagos a la fragmentación del bosque en Pozuzo, Perú
}

\author{
Responses of bats to forest fragmentation at Pozuzo, Peru
}

\author{
José Luis Mena
}

\begin{abstract}
Museo de Historia Natural, Facultad de Ciencias Biológicas, Universidad Ricardo Palma, Av. Benavides 5440, Santiago de Surco, Lima 33, Perú. Lima, Perú.

Email: menaa.jl@gmail.com
\end{abstract}

$\begin{array}{ll}\text { Presentado: } & 08 / 06 / 2010 \\ \text { Aceptado: } & 30 / 11 / 2010\end{array}$

Publicado online: 21/01/2011

\begin{abstract}
Resumen
La deforestación y la fragmentación de los bosques son unas de las amenazas más importantes para la supervivencia de los murciélagos en el Perú. Sin embargo, se conoce muy poco sobre el impacto de estas en sitios o lugares por encima de los $500 \mathrm{~m}$ de altitud. En este estudio, considerando la escala de paisaje, analicé las respuestas de los murciélagos (Chiroptera) a la fragmentación en un paisaje perturbado en Pozuzo (Región Pasco). En ese sentido, considerando el rol de los murciélagos como indicadores de perturbación del hábitat, planteé dos hipótesis. Una primera predicción se refirió a que muestras de paisajes altamente fragmentados y con mayor cantidad de bordes presentarán una mayor abundancia de especies frugívoras que prosperan en hábitats perturbados (Stenodermatinae y Carollinae). La segunda predicción se refería a que muestras de paisaje con una mayor cobertura de bosque deberían tener una mayor abundancia de murciélagos del gremio animalívoro (Phyllostominae), ya que estos son sensibles a las perturbaciones y suelen ser más abundantes en bosques maduros y en buen estado de conservación. Encontré evidencia apoyando la predicción sobre el gremio animalívoro pero apoyo parcial para la predicción sobre los frugívoros. Se resalta la importancia de la conservación de los fragmentos de bosque para asegurar la supervivencia de los murciélagos y de los servicios que estos prestan, en especial para la regeneración del bosque en paisajes tropicales modificados por las actividades humanas.
\end{abstract}

Palabras claves: murciélagos; fragmentación; bosques tropicales; bosque siempreverde subandino del suroeste de la Amazonía; paisajes modificados por el hombre; conservación; Pozuzo; Perú.

\section{Abstract}

Forest fragmentation and deforestation are among the major threats to Peruvian bats conservation. Unfortunately, information about the effects of these threats above $500 \mathrm{~m}$ elevation is lacking. In this study, I assessed bat responses to fragmentation in Pozuzo (Pasco) at a landscape scale approach. I evaluate two hypotheses regarding the role of bats as indicators of habitat disturbance. The first prediction says that landscapes highly disturbed will show higher abundances of habitat generalist species such as frugivorous bats belonging to the subfamilies Stenodermatinae and Carollinae. The second prediction regards that landscapes with greater forest cover will show higher abundance of habitat specialist species such as animalivorous bat species belonging to the subfamily Phyllostominae, a guild sensitive to forest disturbance. I found evidence supporting the animalivorous hypothesis but it was partial to the frugivorous hypothesis. This study highlights the importance of forest fragments to bat conservation in human-modified landscapes.

Keywords: bats; fragmentation; tropical forests; Southwestern Amazon subandean evergreen forest; humanmodified landscapes; conservation; Pozuzo; Peru.

\section{Introducción}

A nivel mundial los bosques tropicales están desapareciendo rápidamente debido a la deforestación y al cambio de uso de la tierra, causando así una tendencia general de reemplazo de los bosques por tierras agrícolas y pastizales para ganado y acoplados con una progresiva pérdida de hábitat y fragmentación (Noss et al. 2006). En cuanto a la fragmentación, su impacto sobre la biodiversidad debe ser analizado a partir del entendimiento de esta perturbación como un proceso, el cual implica cuatro efectos: la reducción en la cantidad del hábitat, el incremento en el número de parches de hábitat, la disminución en el tamaño de los parches y el incremento en el aislamiento de los parches (Fahrig 2003).

La deforestación y la fragmentación producen modificaciones en la disponibilidad y configuración del hábitat, a los cuales las especies pueden o no ajustarse. Por ejemplo, una parte importante de la diversidad de aves terrestres nativas presentes en los bosques tropicales suelen utilizar los campos agrícolas que han surgido luego de la deforestación (Perfecto et al. 2003). Similares resultados han sido encontrados para artrópodos (Perfecto, et al. 2003, Schonberg et al. 2004), algunos grupos de mamíferos terrestres (Daily et al. 2003, Mena \& Medellín 2010, NaughtonTreves et al. 2003) y en murciélagos (Castro-Luna et al. 2007, Medellín et al. 2000).
En general, el impacto de la fragmentación incluye cambios en la diversidad y la abundancia, la dinámica del bosque, la estructura trófica y otros procesos ecológicos y debe ser analizado en la escala de paisaje (Fahrig 2003, Laurance \& Bierregaard 1997). Así, mientras que en la escala de parche se incluyen parámetros como el área, la diversidad de plantas y la estructura de la vegetación; en la escala de paisaje son importantes otros factores tales como el aislamiento y la proximidad entre los tipos de hábitat. Por ejemplo, estudios realizados en la escala de paisaje, revelan la importancia de la cobertura de bosque, el tamaño de los parches y la configuración del paisaje en la diversidad de aves (Graham \& Blake 2001). Además, se tienen evidencias de la importancia del uso de diferentes escalas para entender mejor la respuesta de los murciélagos a la fragmentación (Pinto \& Keitt 2008).

Debido a su abundancia local, riqueza de especies y diversidad ecológica, los murciélagos han sido reconocidos como un grupo indicador de la perturbación antropogénica (Fenton et al. 1992, Medellín et al. 2000, Wilson et al. 1996). De hecho, algunos estudios revelan que en los fragmentos de bosque tropical, la diversidad y abundancia de murciélagos es influenciada por la distancia entre los parches, las estrategias de forrajeo y el tamaño del espacio vital (Cosson et al. 1999, Estrada et al. 1993). Sin em- 

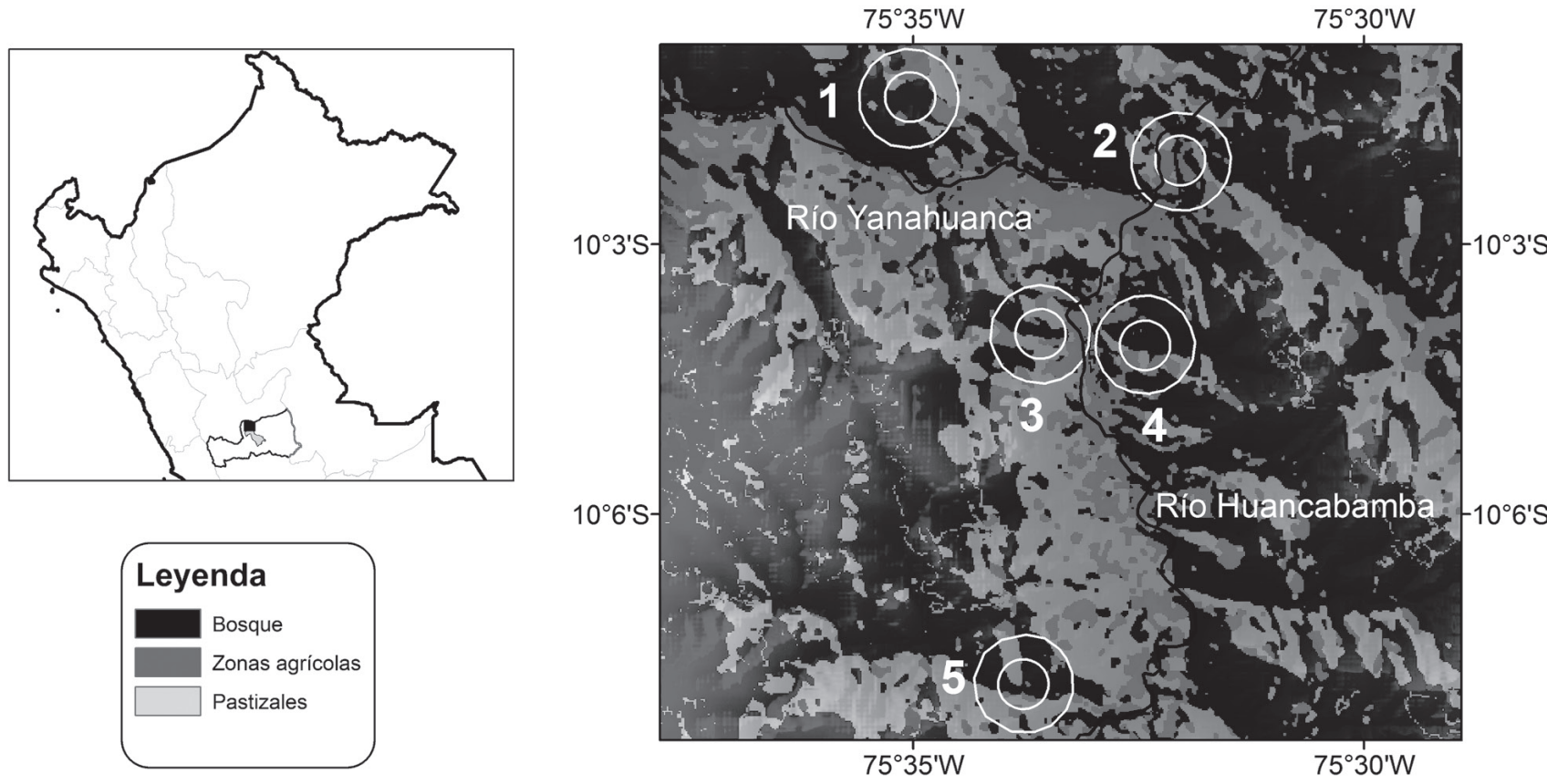

Figura 1. Clasificación de la cobertura vegetal del área de estudio, basado en un imagen de satélite LandSat TM (Septiembre 1997), en donde se puede apreciar los bosques remanentes, pastizales de ganado y el arreglo de las muestras de paisaje: Yanahuanca (1), Sereno-Toropampa (2), Río Negro (3), Palmira (4) y Delfín (5).

bargo, otros estudios revelan que si bien algunas especies suelen usar campos agrícolas y vegetación secundaria, también prefieren ubicar sus sitios de descanso en el bosque maduro (Evelyn \& Stiles 2003), resaltando así el rol de los bosques remanentes en la conservación de estos mamíferos, especialmente en los paisajes rurales tropicales formados a partir de la deforestación y el reemplazo de bosques por áreas agrícolas y ganaderas.

El Perú es uno de los países en Sudamérica que cuenta con mayor extensión de bosques primarios pero también con pérdidas importantes debido a deforestación (FAO 2010). Desafortunadamente, existen pocos estudios que hayan evaluado el impacto de la deforestación o fragmentación sobre la diversidad de murciélagos o la respuesta de ellos ante las perturbaciones antropogénicas (excepto Wilson et al. 1996); aunque recientemente se ha analizado la respuesta de los murciélagos a la fragmentación del bosque tropical amazónico (Klingbeil \& Willig 2009), no se cuenta con información de otras áreas, especialmente en altitudes de la vertiente oriental por encima de los $500 \mathrm{~m}$ de altitud.

El objetivo de este estudio es analizar como los murciélagos responden a la fragmentación del bosque siempreverde subandino del suroeste de la Amazonía, uno de los sistemas ecológicos más diversos pero poco estudiados (Josse et al. 2007). Para este propósito definí dos hipótesis de trabajo que se basaron en varios hallazgos de la respuesta de los murciélagos a las perturbaciones humanas. Por ejemplo, varios estudios han demostrado que por sus requerimientos de dieta, de refugios y hábitat de forrajeo, algunas especies de la subfamilia Stenodermatinae o Carollinae se benefician con cierto grado de perturbación y son abundantes en la vegetación secundaria (Castro-Luna, et al. 2007, Galindo-Leal 2004, Medellín, et al. 2000, Schulze et al. 2000). Por otro lado, los murciélagos de la subfamilia Phyllostominae (Phyllostomidae) y en especial aquellos del gremio animalívoro (aquellos que consumen vertebrados e invertebrados, ver Stevens 1996) suelen ser muy sensibles a las perturbaciones de hábitat y son más abundantes en los bosques maduros (Castro-Luna et al. 2007, Wilson et al. 1996). En este sentido, en la escala de paisaje se puede esperar que muestras de paisajes altamente fragmentados y con un gran efecto de borde deban presentar una mayor abundancia de especies frugívoras oportunistas (principalmente de los géneros Sturnira o Carollia) (Hipótesis 1). Igualmente, se puede predecir una relación positiva entre la cobertura de bosque y la abundancia de murciélagos animalívoros; de este modo, muestras de paisaje con una mayor cobertura de bosque deberían tener una mayor abundancia de estos murciélagos (Hipótesis 2). Complementariamente incluí análisis similares con los otros gremios presentes en la comunidad y con las abundancias de las especies en forma individual, además de una descripción general de la comunidad de murciélagos de Pozuzo.

\section{Materiales y métodos}

\section{Área de estudio}

El estudio se realizó en el valle de Pozuzo (Provincia de Oxapampa, Región Pasco) (Fig. 1), el cual está caracterizado por la presencia de zonas antrópicas y remanentes de bosque siempreverde subandino del suroeste de la Amazonía. Oxapampa es ampliamente reconocida por su biodiversidad y cuenta con dos áreas protegidas muy importantes: el Parque Nacional Yanachaga Chemillén y la Reserva Comunal Yanesha. La precipitación anual promedio de Pozuzo es aproximadamente de $2300 \mathrm{~mm}$, con una estación lluviosa entre los meses de noviembre y abril, y una estación seca entre los meses de mayo y octubre. La temperatura es más o menos uniforme a lo largo del año, con un promedio anual de $22,6{ }^{\circ} \mathrm{C}$. En Pozuzo, predomina un paisaje caracterizado principalmente por actividades humanas, en el cual los bosques remanentes ocurren en fragmentos pequeños o parches (<50 ha) a lo largo de las zonas de pendientes y en fragmentos grandes (> $100 \mathrm{ha}$ ) localizados principalmente en las zonas más alejadas de los centros poblados, ambos inmersos en una matriz de pastizales para ganado y tierras agrícolas (Fig. 
Tabla 1. Localidades y esfuerzo de captura en las localidades evaluadas en Pozuzo (Total de metros de red por hora: $\mathrm{M} \times \mathrm{H}$ ). También se incluye el número esperado de especies según el modelo de Chao y Jacknife (En paréntesis se presenta el porcentaje respecto a lo observado en cada localidad).

\begin{tabular}{lcccccccc}
\hline Localidad & Metros de red & Horas & Noches & M x H & Capturas & \# especies & Chao2 & Jack 2 \\
\hline Delfín & 168 & 28 & 7 & 4704 & 35 & 15 & $20,1(74)$ & $26,2(57)$ \\
Palmira & 216 & 24 & 6 & 5184 & 135 & 27 & $31,2(87)$ & $37,7(72)$ \\
Río Negro & 120 & 20 & 5 & 2400 & 32 & 11 & $15,2(73)$ & $17,9(61)$ \\
Sereno-Toropampa & 144 & 24 & 6 & 3456 & 29 & 13 & $45,0(29)$ & $24,5(53)$ \\
Yanahuanca & 216 & 24 & 6 & 5184 & 25 & 16 & $25,2(64)$ & $30,4(53)$ \\
TOTAL & $\mathbf{8 6 4}$ & $\mathbf{1 2 0}$ & $\mathbf{3 0}$ & $\mathbf{2 0 9 2 8}$ & $\mathbf{2 5 6}$ & $\mathbf{4 2}$ & & \\
\hline
\end{tabular}

1). En general, los bosques remanentes presentan un dosel compuesto principalmente de especies de las familias Bombacaceae, Burseraceae, Combretaceae, Euphorbiaceae, Fabaceae, Meliaceae, Moraceae, Polygonaceae, Sapindaceae, Boraginaceae y Arecaceae; un sotobosque con representantes de Melastomataceae y Piperaceae; y un estrato herbáceo dominado por Araceae, Cyclanthaceae y Heliconaceae. Los pastizales para ganado están caracterizados por la dominancia del pasto exótico Brachiaria decumbens con algunos árboles asociados; mientras que las tierras agrícolas incluyen cultivos como la yuca, el plátano, la papaya, el maíz, entre otros.

\section{Estimación de la diversidad y abundancia de murciélagos}

Entre febrero y agosto de 1996 evalúe 5 localidades (Fig. 1): Delfín (1200 m de altitud), Palmira (900 m), Río Negro (900 $\mathrm{m})$, Sereno-Toropampa $(1000 \mathrm{~m})$ y Yanahuanca $(1200 \mathrm{~m})$. En cada localidad se colocaron de dos a tres redes de neblina a nivel del suelo ( $3 \times 12 \mathrm{~m}$, ojo de malla de $30 \mathrm{~mm}$ ), las cuales fueron abiertas desde las 18:00 hasta las 00:00 horas. Se registró el número de metros extendidos y el tiempo en horas que las redes estuvieron abiertas durante cada noche de trabajo. Las redes fueron ubicadas siempre sobre cursos de agua, dentro del bosque, en los bordes del bosque y cerca de los caminos. Luego de la captura se determinó en cuanto fue posible la especie, además se tomaron datos morfológicos externos, el peso corporal y su condición reproductiva. Una parte de los individuos capturados fueron colectados y depositados en la colección de mamíferos del Museo de Historia Natural, de la Universidad Nacional Mayor de San Marcos (MUSM). Para la determinación de las especies se reviso especímenes del MUSM, además de revisiones taxonómicas para la identificación definitiva del material examinado (Pacheco \& Patterson 1991, Simmons \& Voss 1998, Velazco 2005). La nomenclatura adoptada en este trabajo se basa en Pacheco et al. (2009), la cual sigue la taxonomía de Wilson y Reeder (2005) y Gardner (2008).

Para cuantificar el esfuerzo de captura se obtuvo para cada localidad el producto del total de metros de red (sumando los de cada noche de evaluación) por el total de horas trabajadas (Medellín 1993), lo cual permitió obtener el total de metros de red por hora $(\mathrm{M} \times \mathrm{H})$ en cada localidad (Tabla 1). Este valor ( $\mathrm{M}$ $\mathrm{x} H$ ) sirvió para estimar la abundancia relativa por especie o por gremio, al dividir el número de animales capturados entre $\mathrm{Mx}$ $\mathrm{H}$ (\# de murciélagos por metro de red por hora).

Se elaboraron curvas de acumulación de especies considerando el número de especies capturadas y el número total de capturas para evaluar la calidad del inventario (Soberón \& Llorente 1993). Siguiendo las recomendaciones de Colwell y Coddington (1994) las curvas fueron afinadas por medio de eventos aleatorios (100 veces) para cada localidad y para el total de especies capturadas en Pozuzo. Para cuantificar la totalidad del inventario se usaron los estimadores no paramétricos Chao2 y Jackniffe 2 en los datos de capturas, los cuales fueron estimados con el programa EstimateS (Colwell 2005). Finalmente, se estimó la riqueza de especies esperada en Pozuzo basado en el modelo de Clench (Soberón \& Llorente 1993). La caracterización de los gremios se basó en Kalko y Handley (2001) y Sampaio et al. (2003). Bajo estas definiciones, se identificaron ocho gremios, los cuales se encuentran compuestos por especies que forrajean en hábitats equivalentes y consumiendo alimentos similares (ver Tabla 2). Como animalívoros se agrupa a los gremios de insectívoros y carnívoros acechadores de espacios muy densos (Stevens 1996).

\section{Análisis de paisaje}

La medición de las características del paisaje se basó en una clasificación no supervisada de la cobertura del suelo a partir del análisis de una imagen LandSat TM (septiembre de 1997) con el programa ArcGIS 9.2 (Environmental Systems Research Institute Inc, Redlands, CA). Para motivos de éste análisis se identificaron sólo dos clases: bosque y no bosque (matriz). En términos generales, la estructura del paisaje se caracteriza por su composición y por su configuración y ambos aspectos pueden afectar los procesos ecológicos y a las especies (Bissonette 1997). La composición comprende a la variedad y la abundancia de parches dentro de un paisaje. De hecho, muchas especies de vertebrados requieren de tipos de hábitat específicos (p.ej. bosques), de modo tal que la cantidad total de dicho hábitat

Tabla 2. Definición de los gremios presentes en Pozuzo de acuerdo a Kalko y Handley (2001) y Sampaio (2003).

\begin{tabular}{clc}
\hline Gremios & \multicolumn{1}{c}{ Descripción } & $\begin{array}{c}\text { N.o de } \\
\text { especies }\end{array}$ \\
\hline I & $\begin{array}{l}\text { Insectívoros aéreos de espacios } \\
\text { abiertos }\end{array}$ & 2 \\
II & $\begin{array}{l}\text { Insectívoros aéreos de espacios de } \\
\text { fondo denso }\end{array}$ & 6 \\
IV & $\begin{array}{l}\text { Insectívoros acechadores de espacios } \\
\text { muy densos }\end{array}$ & 4 \\
V & $\begin{array}{l}\text { Carnívoros acechadores de espacios } \\
\text { muy densos }\end{array}$ & 1 \\
VII & $\begin{array}{l}\text { Hematófagos acechadores de espacios } \\
\text { muy densos }\end{array}$ & 1 \\
VIII & $\begin{array}{l}\text { Frugívoros acechadores de espacios } \\
\text { muy densos }\end{array}$ & 25 \\
IX & $\begin{array}{l}\text { Nectarívoros acechadores de espacios } \\
\text { muy densos }\end{array}$ & 5 \\
X & $\begin{array}{l}\text { Omnívoros acechadores de espacios } \\
\text { muy densos }\end{array}$ & 2 \\
\hline
\end{tabular}




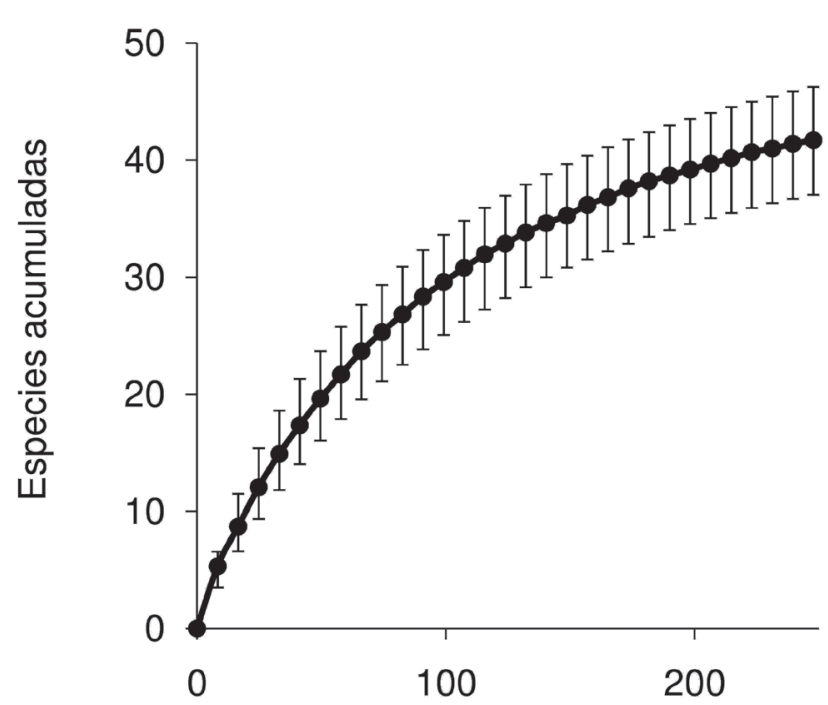

Capturas acumuladas

Figura 2. Curva de acumulación de especies basado en el número de murciélagos capturados en Pozuzo.

probablemente influencie su ocurrencia y abundancia. Por otro lado, la configuración del paisaje se refiere a la distribución física o al arreglo espacial de los parches. En cada localidad (5) se seleccionaron dos muestras circulares de paisaje, una de 500 $\mathrm{m}$ y otra de $1000 \mathrm{~m}$ de radio (lo cual permitió evaluar alguna asociación que sea dependiente de la escala). La elección de estas dos escalas se basó en lo sugerido en estudios previos (Gorresen \& Willig 2004, Klingbeil \& Willig 2009). Cada muestra de paisaje fue caracterizada con base en su composición y configuración, considerando las variables descritas en Gorresen y Willig (2004) y Klingbeil y Willig (2009). Así, las variables que caracterizaron la composición del paisaje fueron el porcentaje de la cobertura de bosque (COVER) y la densidad de parches (PD). Mientras que las variables que caracterizaron la configuración fueron la densidad de borde (ED) y el promedio del índice de la forma

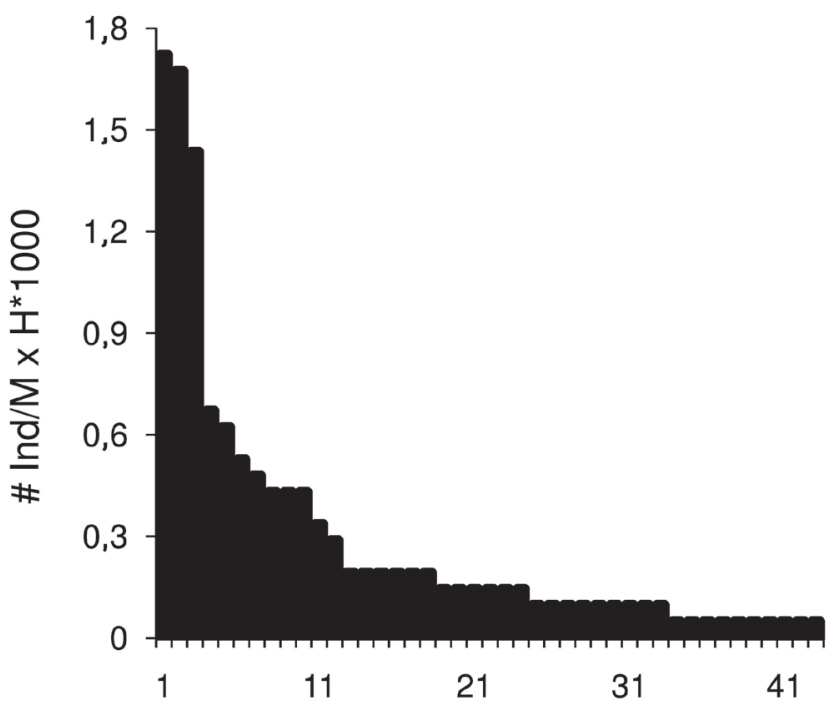

\section{Especies}

Figura 3. Histograma de abundancias de las especies de murciélagos capturados en Pozuzo, basado en el número de individuos por metro por hora. Para una mejor apreciación los valores están multiplicados por mil. de los parches (SHAPE). El valor de estas variables fue obtenido a través de un análisis realizado con el programa Fragstat 3.3 (McGarigal et al. 2002).

\section{Análisis estadístico}

La existencia de una relación entre las variables que caracterizaron la estructura del paisaje con la riqueza de especies, la abundancia de cada especie y la abundancia de los gremios tróficos $(\mathrm{N}=5)$ fue analizada con la prueba no paramétrica de Spearman. Esta prueba es útil con tamańos pequeños de muestra y cuando se desconoce la distribución de los datos; de modo que nos permite determinar si existe una relación monotónica entre dos variables (Ramsey \& Schafer 2002). Con esta prueba también se analizó la existencia de correlación entre las variables que caracterizan el paisaje. Estos análisis se realizaron en el programa R 2.10.1 (R Development Core Team 2005).

\section{Resultados}

El muestreo comprendió un total de $864 \mathrm{~m}$ de red con 120 horas trabajadas durante 30 noches (Tabla 1). Se capturaron 256 individuos que correspondieron a 42 especies, 23 géneros y 4 familias (Apéndice 1). La familia con más especies fue Phyllostomidae (38 especies). Entre los Phyllostomidae, la subfamilia Stenodermatinae (21 especies) fue la más diversa. Palmira fue la localidad donde se registró el mayor número de especies y fue la mejor muestreada según los estimadores Chao2 y Jackniffe2, seguidos de Delfín y Río Negro (Tabla 1). En total, en las 30 noches de trabajo se colectó el $76 \%$ de las especies esperadas para Pozuzo según el modelo de Clench (58 especies). Además, la curva de acumulación de las especies capturadas en Pozuzo, sugiere que ésta se aproxima a una asíntota (Fig. 2).

El histograma de abundancia relativa (Fig. 3) revela que existen tres especies dominantes en Pozuzo (Carollia brevicauda, Carollia perspicillata y Platyrrhinus infuscus), las cuales representan cerca del $40 \%$ del total de capturas. En cuanto a los gremios tróficos, los frugívoros acechadores de fondo denso fueron el gremio más diverso (54\% del total de especies), seguidos de los insectívoros aéreos de espacios de fondo denso (13\%) y de los nectarívoros (11\%). Pozuzo está caracterizado por especies típicas de tierras bajas como Chiroderma trinitatum, Micronycteris minuta y Thyroptera tricolor, así como, de especies de las vertientes orientales de los Andes como Platyrrhinus albericoi, P. masu, P. nigellus y Sturnira erythromos.

Se encontraron relaciones dependientes de la escala entre la abundancia de los murciélagos y la estructura del paisaje. Es decir, algunas variables son importantes en una escala pero no en la otra (Tabla 3). La relación entre la abundancia de Carollia benkeithi y la densidad de borde es la única que provee soporte a la hipótesis 1 . Se encontró una relación positiva entre la cobertura de bosque con la abundancia del gremio IV (murciélagos insectívoros acechadores de espacios altamente densos: Micronycteris hirsuta, M. megalotis, M. minuta y Tonatia saurophila) y los animalívoros (Gremios IV y V), lo cual provee soporte a la hipótesis 2 .

\section{Discusión}

A pesar de ser una zona altamente perturbada y además ubicada por encima de los $800 \mathrm{~m}$ de altitud, la diversidad de murciélagos de Pozuzo es realmente destacable. En total se documentan 46 especies incluyendo registros adicionales reportados 
Tabla 3. Relaciones entre características del paisaje con las especies y gremios de murciélagos de Pozuzo. COVER: porcentaje de la cobertura de bosque, PD: la densidad de parches, ED: densidad de borde y SHAPE: promedio del índice de la forma de los parches. Los valores de significancia corresponden a la prueba de Spearman.

\begin{tabular}{|c|c|c|c|c|c|}
\hline \multirow[b]{2}{*}{ Variable } & \multirow[b]{2}{*}{ Escala } & \multicolumn{2}{|c|}{ COMPOSICION } & \multicolumn{2}{|c|}{ CONFIGURACION } \\
\hline & & COVER & PD & ED & SHAPE \\
\hline \multirow{2}{*}{ Artibeus glaucus } & $1000 \mathrm{~m}$ & $0,89^{*}$ & $-0,89 *$ & ns & ns \\
\hline & $500 \mathrm{~m}$ & ns & ns & $-0,89^{*}$ & $-0,89 *$ \\
\hline \multirow[t]{2}{*}{ Artibeus obscurus } & $1000 \mathrm{~m}$ & ns & ns & ns & $0,89^{*}$ \\
\hline & $500 \mathrm{~m}$ & ns & $\mathrm{ns}$ & ns & ns \\
\hline \multirow{2}{*}{ Carollia benkeithi } & $1000 \mathrm{~m}$ & ns & ns & $0,89^{*}$ & ns \\
\hline & $500 \mathrm{~m}$ & ns & ns & ns & ns \\
\hline \multirow[t]{2}{*}{ Carollia brevicauda } & $1000 \mathrm{~m}$ & ns & $\mathrm{ns}$ & ns & ns \\
\hline & $500 \mathrm{~m}$ & ns & ns & ns & $0,97^{* *}$ \\
\hline \multirow[t]{2}{*}{ Sturnira spp. } & $1000 \mathrm{~m}$ & $1,00^{*}$ & ns & ns & ns \\
\hline & $500 \mathrm{~m}$ & ns & $\mathrm{ns}$ & $-1,00^{*}$ & ns \\
\hline \multirow[t]{2}{*}{ Carollia spp. } & $1000 \mathrm{~m}$ & ns & ns & ns & ns \\
\hline & $500 \mathrm{~m}$ & ns & $-0,89 *$ & ns & ns \\
\hline \multirow[t]{2}{*}{ Gremio II } & $1000 \mathrm{~m}$ & ns & ns & ns & ns \\
\hline & $500 \mathrm{~m}$ & ns & $-0,89 *$ & ns & ns \\
\hline \multirow[t]{2}{*}{ Gremio IV } & $1000 \mathrm{~m}$ & $1,00^{*}$ & ns & ns & ns \\
\hline & $500 \mathrm{~m}$ & ns & ns & $-1,00^{*}$ & ns \\
\hline \multirow{2}{*}{ Animalívoros } & $1000 \mathrm{~m}$ & $1,00^{*}$ & ns & ns & ns \\
\hline & $500 \mathrm{~m}$ & ns & ns & $-1,00^{*}$ & ns \\
\hline
\end{tabular}

en Solari et al. (1999) y capturas fuera de los eventos de muestreo (Apéndice 1). Es más, según el modelo de Clench se podría esperar hasta 12 especies más. En efecto, la composición de especies es muy similar a la registrada en altitudes similares del Parque Nacional Yanachaga Chemillén con 47 especies (Vivar 2006).

En general, los efectos de la fragmentación sobre la diversidad de murciélagos han sido documentados principalmente en bosques de tierras bajas (Bernard \& Fenton 2007, Presley et al. 2009). Sus efectos parecen ser dependientes principalmente de los rasgos particulares a nivel de la especie y también de la escala espacial, tal y como ha sido reportado en algunas especies de murciélagos frugívoros de la familia Phyllostomidae (Pinto \& Keitt 2008). En Pozuzo, la respuesta de Carollia benkeithi a la fragmentación es concordante con lo esperado para las especies de éste género, las cuales se alimentan principalmente de las frutas de Piper (Piperaceae), prosperando en bosques perturbados y en vegetación secundaria (de Lima \& dos Reis 2004, Giannini \& Kalko 2004). Efectivamente, algunas de las especies de murciélagos frugívoros responden significativamente a las características de la composición y configuración del paisaje, incrementando su abundancia en sitios moderadamente fragmentados, explotando densidades elevadas de recursos de alimento después de la conversión del bosque a campos agrícolas y también durante la subsecuente sucesión (Klingbeil \& Willig 2009).

Varios estudios han demostrado que la mortalidad de árboles y la formación de claros en el dosel se incrementan cerca de los bordes, lo cual modifica la estructura de los gremios y la composición de plantas leñosas (Laurance et al. 2006). Estos cambios implican la pérdida de hábitat de forrajeo y refugio para algunas especies de murciélagos. Por ejemplo, el murciélago frugívoro Artibeus glaucus, que al igual que otras especies pequeñas de $A r-$ tibeus, suelen usar principalmente el dosel del bosque (Peters et al. 2006, Sampaio et al. 2003) sería una especie potencialmente afectada, como parece ser el caso en Pozuzo. La abundancia de $A$. glaucus en la escala de $1000 \mathrm{~m}$, parece estar relacionada positivamente con una mayor cobertura de bosque y en escalas menores esta parece estar afectada negativamente por la densidad de borde y la densidad de parches de bosque (ver Tabla 3). En Perú, esta especie ha sido principalmente capturada en bosques primarios (Hice et al. 2004) aunque también en áreas perturbadas (Pacheco et al. 2007) Probablemente, el pequeño tamaño de esta especie es una limitante para volar grandes distancias entre los fragmentos de bosque.

En áreas con bajos niveles de deforestación, los murciélagos frugívoros suelen cruzar las áreas abiertas o perturbadas en búsqueda de alimento y refugio en los fragmentos de bosque (Bernard \& Fenton 2007, Morrison 1980). Sin embargo, con el incremento de las áreas deforestadas, la conectividad de los parches de bosque remanentes disminuye y probablemente pocas especies de frugívoros podrían ser capaces de cruzar las grandes áreas sin cobertura de bosque (Klingbeil \& Willig 2009). Por ejemplo, algunos estudios sugieren que la fragmentación influye negativamente en la abundancia de Artibeus obscurus (Faria 2006), aunque otros estudios sugieren que esta especie parece ser menos sensible a la fragmentación que otras especies de Artibeus grandes (Henry et al. 2007) o simplemente no es afectada (Bernard \& Fenton 2007). En efecto, A. obscurus fue uno de los frugívoros más abundantes en Pozuzo, tal y como ha sido reportado en otros paisajes fragmentados por la acción del hombre (Bernard \& Fenton 2007, Cosson et al. 1999).

En cuanto a los murciélagos animalívoros, estos han sido reconocidos como un grupo sensible al efecto de borde y responden negativamente a la perturbación y a la fragmentación (Medellín, et al. 2000, Wilson, et al. 1996). No obstante, en algunos casos parecen ser favorecidos por la perturbación del hábitat (Klingbeil \& Willig 2009). En Pozuzo, los resultados son más consistentes con la hipótesis de sensibilidad a la fragmentación y a la perturbación del hábitat. Estos murciélagos son sensibles a los cambios en la estructura de los bosques (Clarke et al. 2005, Jiménez-Ortega \& Mantilla-Meluk 2008), además están morfológicamente limitados a vuelos de corta distancia, cuentan con ámbitos hogareños reducidos, con sitios de forrajeo a menudo localizados en el interior del bosque (Bernard \& Fenton 2007, Kalko et al. 1999). Al parecer, estos murciélagos 
son capaces de sobrevivir en paisajes altamente fragmentados sólo si el grado de aislamiento de los remanentes de bosque es bajo y si hay proximidad espacial a los bosques continuos más grandes (Meyer et al. 2008).

En los últimos años, la conservación fuera de las áreas protegidas ha tomado gran importancia, en la medida que se ha reconocido que las áreas naturales protegidas son por lo general insuficientes para conservar la mayor parte de la biodiversidad. Fuera de las áreas protegidas, las especies nativas pueden continuar viviendo en hábitats que, aunque sujetos a actividades productivas, mantienen la estructura y las funciones básicas de sus ecosistemas originales. Este último es el caso de los murciélagos. Por ejemplo, Artibeus obscurus y otras especies de murciélagos frugívoros como Carollia perspicillata y Sturnira lilium, funcionan como especies clave para el bosque tropical por su rol en la dispersión de semillas y cualquier impacto negativo en sus poblaciones provocarían serios problemas a la conservación y regeneración del bosque en paisajes fragmentados (Aguiar \& Marinho-Filho 2007, de Lima \& dos Reis 2004). En este caso, la conservación de poblaciones saludables de murciélagos en los bosques continuos y en los fragmentos de bosque son fundamentales para asegurar que los servicios que prestan estas especies puedan mantenerse (p.ej. dispersión de semillas o polinización). Desafortunadamente, con los incrementos de la población humana en zonas rurales, la tendencia a la ampliación de la frontera agrícola es cada vez mayor. Por esta razón, es importante conocer la ecología de las especies en los bosques fragmentados, lo cual permitirá contar con información valiosa para el diseño de estrategias de conservación en este tipo de paisajes. Este estudio contribuye con información original sobre el uso de hábitat por los murciélagos en paisajes tropicales modificados por las actividades humanas por encima de los 500 $\mathrm{m}$ de altitud, la cual puede ser reevaluada posteriormente, para conocer el impacto de la fragmentación en la supervivencia de estos mamíferos en el mediano y en el largo plazo.

\section{Agradecimientos}

Este estudio conto con el apoyo de la ONG PROTERRA. Agradezco de manera especial a todos los asistentes que colaboraron en la evaluación de campo. Al entonces Instituto Nacional de Recursos Naturales por la emisión de los permisos correspondientes para la investigación en Pozuzo. A Sergio Solari por su apoyo en la determinación de los especímenes y asesoramiento para las evaluaciones de campo. A Juan Carlos Riveros por sus valiosos comentarios a una versión anterior de este manuscrito. Armando Mercado colaboró con la clasificación de la imagen satelital de Pozuzo. Al Departamento de Mastozoología del Museo de Historia Natural (MUSM) de la Universidad nacional mayor de San Marcos por su apoyo con el equipo de campo y por brindarme las facilidades para la revisión de los especímenes y la base de datos de la colección de mamíferos.

\section{Literatura citada}

Aguiar L.M.S. \& J. Marinho-Filho. 2007. Bat frugivory in a remnant of Southeastern Brazilian Atlantic forest. Acta Chiropterologica 9: 251-260.

Bernard E. \& M.B. Fenton. 2007. Bats in a fragmented landscape: Species composition, diversity and habitat interactions in savannas of Santarem, Central Amazonia, Brazil. Biological Conservation 134: 332-343.

Bissonette J.A. 1997. Wildlife and landscape ecology: effects of pattern and scale. Springer-Verlag.
Castro-Luna A.A., V.J. Sosa \& G. Castillo-Campos. 2007. Quantifying phyllostomid bats at different taxonomic levels as ecological indicators in a disturbed tropical forest. Acta Chiropterologica 9: 219-228.

Clarke F.M., L.V. Rostant \& P.A. Racey. 2005. Life after logging: post-logging recovery of a neotropical bat community. Journal of Applied Ecology 42: 409-420.

Colwell R.K. 2005. EstimateS: statistical estimation of species richness and shared species from samples, Ver 7.5.1. Persistent URL $<$ purl.oclc.org/estimates $>$.

Colwell R.K. \& J.A. Coddington. 1994. Estimating terrestrial biodiversity through extrapolation. Philosophical Transactions of the Royal Society B. Biological Sciences 345: 101-118.

Cosson J.-F., J.-M. Pons \& D. Masson. 1999. Effect of forest fragmentation on frugivorous and nectarivorous bats in French Guiana. Journal of Tropical Ecology 15: 515-534.

Daily G.C., G. Ceballos, J. Pacheco, et al. 2003. Countryside biogeography of Neotropical mammals: conservation opportunities in agricultural landscapes of Costa Rica. Conservation Biology 17: 1814-1826.

de Lima, I.P. \& N.R. dos Reis. 2004. The availability of Piperaceae and the search for this resource by Carollia perspicillata (Linnaeus) (Chiroptera, Phyllostomidae, Carolliinae) in Parque Municipal Arthur Thomas, Londrina, Paraná, Brazil. Revista Brasileira de Zoologia 21: 371.

Estrada A., R. Coates-Estrada \& J.D. Merritt. 1993. Bat species richness and abundance in tropical rain forest fragmentsand in agricultural habitats at Los Tuxtlas, Mexico. Ecography 16: 309-318.

Evelyn M.J. \& D.A. Stiles. 2003. Roosting requirements of two frugivorous bats (Sturnira lilium and Artibeus intermedius) in fragmented Neotropical forest. Biotropica 35: 405-418.

Fahrig L. 2003. Effects of Habitat Fragmentation on Biodiversity. Annual Review of Ecology, Evolution, and Systematics 34: 487-515.

FAO. 2010. Evaluación de los recursos forestales mundiales 2010: Informe principal.

Faria D. 2006. Phyllostomid bats of a fragmented landscape in the north-eastern Atlantic forest, Brazil. Journal of Tropical Ecology 22: 531-542.

Fenton M.B., L. Acharya, D. Audet, et al. 1992. Phyllostomid bats (Chiroptera: Phyllostomidae) as indicators of habitat disruption in the Neotropics. Biotropica 24: 440-446.

Galindo-Leal C. 2004. Clasificación de los murciélagos de la región de los Tuxtlas, Veracruz, respecto a su respuesta a la fragmentación del hábitat. Acta Zoológica Mexicana (n.s.) 20: 239-243.

Gardner A.L. (ed.). 2008. Mammals of South America, vol. 1: marsupials, xenarthrans, shrews, and bats. University of Chicago Press.

Giannini N.P. \& E.K.V. Kalko. 2004. Trophic structure in a large assemblage of phyllostomid bats in Panama. Oikos 105: 209-220.

Gorresen P.M. \& M.R. Willig. 2004. Landscape responses of bats to habitat fragmentation in Atlantic forest of Paraguay. Journal of Mammalogy 85: 688-697.

Graham C.H. \& J.G. Blake. 2001. Influence of patch and lanscape level factors on bird assemblages in a fragmented tropical landscape. Ecological Applications 11: 1709-1721.

Henry M., J.-F. Cosson \& J.-M. Pons. 2007. Abundance may be a misleading indicator of fragmentation-sensitivity: the case of fig-eating bats. Biological Conservation 139: 462-467.

Hice C.L., P.M. Velazco \& M.R. Willig. 2004. Bats of the Reserva Nacional Allpahuayo-Mishana, northeastern Peru, with notes on community structure. Acta Chiropterologica 6: 319-334. 
Jiménez-Ortega A.M. \& H. Mantilla-Meluk. 2008. El papel de la tala selectiva en la conservación de bosques neotropicales y la utilidad de los murciélagos como bioindicadores de disturbio. Revista Institucional Universidad Tecnológica del Chocó: Investigación, Biodiversidad y Desarrollo 27: 100-108.

Josse C., G. Navarro, F. Encarnación, et al. 2007. Sistemas Ecológicos de la Cuenca Amazónica de Perú y Bolivia. Clasificación y mapeo. NatureServe.

Kalko E.K. \& C.O. Handley, Jr. 2001. Neotropical bats in the canopy: diversity, community structure, and implications for conservation. Plant Ecology 153: 319-333.

Kalko E.K.V., D. Friernel, C.O. Handley, Jr. \& H.U. Schnitzler. 1999. Roosting and foraging behavior of two Neotropical gleaning bats, Tonatia silvicola and Trachops cirrhosus (Phyllostomidae). Biotropica 31: 344-353.

Klingbeil B.T. \& M.R. Willig. 2009. Guild-specific responses of bats to landscape composition and configuration in fragmented Amazonian rainforest. Journal of Applied Ecology 46: 203-213.

Laurance W.F. \& R.O. Bierregaard (eds.). 1997. Tropical Forest Remnants. Ecology, Management, and Conservation of Fragmented Communities. The University of Chicago Press.

Laurance W.F., H.E.M. Nascimento, S.G. Laurance, et al. 2006. Rapid decay of tree-community composition in Amazonian forest fragments. Proceedings of the National Academy of Sciences 103: 19010.

McGarigal K., S.A. Cushman, M.C. Neel \& E. Ene. 2002. FRAGSTATS: spatial pattern analysis program for categorical maps [online]. Disponible en www.umass.edu/landeco/ research/fragstats/fragstats.html [acceso en septiembre del 2009].

Medellín R.A. 1993. Estructura y diversidad de una comunidad de murciélagos en el tropico humedo Mexicano. - En: Medellín, R. A. and Ceballos, G. (eds.), Avances en el Estudio de los Mamferos de Mexico. Publicaciones Especiales. Asociación Mexicana de Mastozoología, A. C., México D. F. Pp. 333-354.

Medellín R.A., M. Equihua \& M.A. Amin. 2000. Bat diversity and abundance as indicators of disturbance in Neotropical rainforests. Conservation Biology 14: 1666-1675.

Mena J.L. \& R.A. Medellín. 2010. Small mammal assemblages in a disturbed tropical landscape at Pozuzo, Peru. Mammalian Biology 75: 83-91.

Meyer C.F.J., J. Fründ, W.P. Lizano \& E.K.V. Kalko. 2008. Ecological correlates of vulnerability to fragmentation in Neotropical bats. Journal of Applied Ecology 45: 381-391.

Morrison D.W. 1980. Foraging and day-roosting dynamics of canopy fruit bats in Panama. Journal of Mammalogy 61: 20-29.

Naughton-Treves L., J.L. Mena, A. Treves, et al. 2003. Wildlife Survival Beyond Park Boundaries: the Impact of Slash-andBurn Agriculture and Hunting on Mammals in Tambopata, Peru. Conservation Biology 17: 1106-1117.

Noss R., B. Custi \& M. J. Groom. 2006. Habitat fragmentation. En: Groom, M. J. et al. (eds.), Principles of Conservation Biology. Sinauer Associates, Inc., Sunderland, USA. Pp. 213-251.

Pacheco V., R. Cadenillas, E. Salas, et al. 2009. Diversidad y endemismo de los mamíferos del Perú. Revista Peruana de Biología 16: 5-32.

Pacheco V. \& B.D. Patterson. 1991. Phylogenetic relationships of the New World bat genus Stunira (Chiroptera: Phyllostomidae). Bulletin of the American Museum of Natural History 206: 101-121.
Pacheco V., E. Salas, L. Cairampoma, et al. 2007. Contribución al conocimiento de la diversidad y conservación de los mamíferos en la cuenca del río Apurímac, Perú. Revista Peruana de Biología 14: 169-180.

Perfecto I., A. Mas, T. Dietsch \& J. Vandermeer. 2003. Conservation of biodiversity in coffee agroecosystems: a tri-taxa comparison in southern Mexico. Biodiversity and Conservation 12: $1239-1252$

Peters S.L., J.R. Malcolm \& B.L. Zimmerman. 2006. Effects of selective logging on bat communities in the southeastern Amazon. Conservation Biology 20: 1410-1421.

Pinto N. \& T. H. Keitt. 2008. Scale-dependent responses to forest cover displayed by frugivore bats. Oikos 117: 1725-1731.

Presley S.J., M.R. Willig, I. Castro-Arellano \& S. C. Weaver. 2009. Effects of habitat conversion on temporal activity patterns of phyllostomid bats in lowland Amazonian rain forest. Journal of Mammalogy 90: 210-221.

Ramsey F.L. \& D.W. Schafer. 2002. The statistical sleuth: A course in methods of data analysis. Duxbury-Thomson Learning. 742

Sampaio E.M., E.K. Kalko, E. Bernard, et al. 2003. A biodiversity assessment of bats (Chiroptera) in a tropical lowland rainforest of Central Amazonia, including methodological and conservation considerations. Studies on Neotropical Fauna and Environment 38: 17-31.

Schonberg L.A., J.T. Longino, N.M. Nadkarni, et al. 2004. Arboreal ant species richness in primary forest, secondary forest, and pasture habitats of a tropical montane landscape. Biotropica 36: 402-409.

Schulze M.D., N.E. Seavy \& D.F. Whitacre. 2000. A comparison of the phyllostomid bat assemblages in undisturbed Neotropical forest and in forest fragments of a slash-and-burn farming mosaic in Petén, Guatemala. Biotropica 32: 174-184.

Simmons N.B. \& R.S. Voss. 1998. The mammals of Paracou, French Guiana: a Neotropical rainforest fauna. Part I: Bats. Bulletin of the American Museum of Natural History 237: 237.

Soberón J.M. \& J. Llorente. 1993. The Use of species accumulation functions for the prediction of species richness. Conservation Biology 7: 480-488.

Solari S., V. Pacheco \& E. Vivar. 1999. New Distribution records of Peruvian bats. Revista Peruana de Biología 6: 152-159.

Stevens R.D. 1996. Ecomorphological structure of bat communities: alternative models and environmental gradients. A thesis submitted to the Graduate Faculty of Texas Tech University in Partial Fulfillment of the Requirements for the Degree of Master of Science.

Velazco P.M. 2005. Morphological phylogeny of the bat genus Platyrrhinus Saussure, 1860 (Chiroptera: Phyllostomidae) with the description of four new species. Fieldiana Zoology 105: 1-53.

Vivar E. 2006. Análisis de distribución altitudinal de mamíferos pequeños en el parque nacional Yanachaga Chemillén, Pasco, Perú. Tesis, Magister en Zoologia, mención Sistemática y Evolución. Facultad de Ciencias Biológicas, Universidad Nacional Mayor de San Marcos, Lima, Perú. 103

Wilson D.E., C.F. Ascorra, S. Solari, D.E. Wilson \& A. Sandoval. 1996. Bats as indicators of habitat disturbance. - En: Manu: The Biodiversity of Southeastern Peru. Smithsonian Institution Press, Lima. Pp. 613-625.

Wilson D.E. \& D.M. Reeder (eds.). 2005. Mammal species of the World. Johns Hopkins University Press. 2142 
Apéndice 1. Lista de especies de murciélagos registrados en Pozuzo. En la columna de peso (en gramos); se señala entre paréntesis la desviación estándar y en la columna de reproducción los meses de febrero (02) y agosto (08). Estado reproductivo L: lactando, P: preñadas. $\mathrm{N}=$ número de individuos y $\mathrm{AB}=$ Antebrazo. ${ }^{*}$ Reportado por Solari et al. (1999). ${ }^{* *}$ Capturas fuera de los eventos de muestreo.

\begin{tabular}{|c|c|c|c|c|}
\hline Nombre científico & $\mathbf{N}$ & Peso (g) & $\mathrm{AB}(\mathrm{mm})$ & Reproducción \\
\hline \multicolumn{5}{|l|}{ PHYLLOSTOMIDAE } \\
\hline \multicolumn{5}{|l|}{ Subfamilia Desmodontinae } \\
\hline 1. Desmodus rotundus (E. Geoffroy, 1810) & 13 & 33,4 & $62,0(4,6)$ & \\
\hline \multicolumn{5}{|l|}{ Subfamilia Glossophaginae } \\
\hline 2. Anoura caudifer (E. Geoffroy, 1818) & 2 & 9,5 & $36,0(1,4)$ & $\mathrm{L}(08)$ \\
\hline 3. Anoura cultrata Handley, 1960 & 2 & 15 & 40,5 & \\
\hline 4. Anoura geoffroyi Gray, 1838 & 9 & 12 & $41,5(4,0)$ & \\
\hline \multicolumn{5}{|l|}{ Subfamilia Lonchophyllinae } \\
\hline 5. Lonchophylla handleyi Hill, 1980 & 2 & & & \\
\hline \multicolumn{5}{|l|}{ 6. Lonchophylla robusta* Miller, 1912} \\
\hline \multicolumn{5}{|l|}{ Subfamilia Phyllostominae } \\
\hline 7. Chrotopterus auritus (Peters, 1856) & 1 & 71 & 81 & \\
\hline 8. Lophostoma silvicolum d'Orbigny, 1836 & 4 & 32,5 & $59,6(0,71)$ & \\
\hline 9. Micronycteris hirsuta (Peters, 1869) & 1 & 13 & 46 & \\
\hline 10. Micronycteris megalotis (Gray, 1842) & 3 & 5 & $33,0(2,7)$ & \\
\hline 11. Micronycteris minuta (Gervais, 1856) & 2 & 7 & 37 & \\
\hline 12. Phylloderma stenops Peters, 1865 & 2 & 54 & $71,5(2,1)$ & $\mathrm{L}(02)$ \\
\hline \multicolumn{5}{|l|}{ 13. Phyllostomus elongatus* (E. Geoffroy, 1810) } \\
\hline 14. Phyllostomus hastatus (Pallas, 1767) & 9 & 94,3 & $88,7(3,1)$ & $\mathrm{P}(02)$ \\
\hline \multicolumn{5}{|l|}{ Subfamilia Carolliinae } \\
\hline 15. Carollia benkeithi Solari y Baker, 2006 & 10 & 11,1 & $37,7(7,1)$ & \\
\hline 16. Carollia brevicauda (Schinz, 1821) & 36 & 14,8 & 40,9 & \\
\hline 17. Carollia perspicillata (Linnaeus, 1758) & 33 & 17,1 & $42,4(1,3)$ & $\mathrm{L}(02)$ \\
\hline \multicolumn{5}{|l|}{ Subfamilia Stenodermatinae } \\
\hline 18. Artibeus anderseni Osgood, 1916 & 3 & 21 & $37,0(1,4)$ & \\
\hline 19. Artibeus cf. cinereus (Gervais, 1856) & 3 & 11 & 40 & \\
\hline 20. Artibeus glaucus Thomas, 1893 & 9 & 12 & $39,2(1,5)$ & \\
\hline 21. Artibeus lituratus (Olfers, 1818) & 4 & 81,5 & $74,0(1,4)$ & \\
\hline 22. Artibeus obscurus (Schinz, 1821) & 11 & 36,7 & $58,5(2,7)$ & $\mathrm{L}(02), \mathrm{P}(02$ y 08$)$ \\
\hline 23. Artibeus planirostris (Spix, 1823) & 7 & 66 & $72,5(2,1)$ & \\
\hline 24. Chiroderma salvini Dobson, 1878 & 3 & 39 & 54 & $\mathrm{P}(02)$ \\
\hline 25. Chiroderma trinitatum Goodwin, 1958 & 1 & & & \\
\hline 26. Enchistenes hartii (Thomas, 1892) & 4 & 14,5 & $41,5(0,7)$ & \\
\hline 27. Mesophylla macconelli Thomas, 1901 & 4 & 8 & $32,7(2,1)$ & \\
\hline 28. Platyrrhinus albericoi Velazco, 2005 & 3 & & & \\
\hline 29. Platyrrhinus incarum (Thomas, 1912) & 2 & & & \\
\hline 30. Platyrrhinus infuscus (Peters, 1880) & 30 & 42,8 & $57,7(1,6)$ & L y $P(02)$ \\
\hline 31. Platyrrhinus masu Velazco, 2005 & 6 & 26 & $50,5(0,8)$ & \\
\hline 32. Platyrrhinus nigellus Gardner y Carter, 1972 & 4 & 19,5 & $45,0(1,4)$ & \\
\hline 33. Sturnira erythromos (Tschudi, 1844) & 1 & 15 & 42 & \\
\hline 34. Sturnira lilium (E. Geoffroy, 1810) & 14 & 20,3 & $43,1(1,7)$ & $\mathrm{L}(02,08), \mathrm{P}(08)$ \\
\hline 35. Sturnira oporaphilum (Tschudi, 1844) & 4 & 20 & 46 & \\
\hline 36. Uroderma bilobatum Peters, 1866 & 1 & 18 & 44 & \\
\hline 37. Vampyressa thyone Thomas, 1909 & 1 & 8 & 32 & \\
\hline 38. Vampyrodes caraccioli (Thomas, 1889) & 3 & 30,7 & $52,7(2,5)$ & \\
\hline \multicolumn{5}{|l|}{ THYROPTERIDAE } \\
\hline 39. Thyroptera tricolor Spix, 1823 & 1 & 5 & 38 & \\
\hline \multicolumn{5}{|l|}{ MOLOSSIDAE } \\
\hline 40. Molossus molossus (Pallas, 1766) & 2 & 16,3 & $36,0(3,6)$ & \\
\hline 41. Molossus rufus E. Geoffroy, 1805 & 1 & 46 & 54 & \\
\hline \multicolumn{5}{|l|}{ VESPERTILIONIDAE } \\
\hline \multicolumn{5}{|l|}{ 42. Eptesicus brasilienis** (Desmarest, 1819) } \\
\hline 43. Lasiurus blossevillii** (Lesson y Garnot, 1826) & & 7 & 41 & \\
\hline 44. Myotis nigricans (Schinz, 1821) & 2 & 5,8 & $36,0(3,5)$ & $\mathrm{L}(02)$ \\
\hline 45. Myotis riparius Handley, 1960 & 2 & 5,5 & $39,0(0,0)$ & \\
\hline 46. Myotis simus Thomas, 1901 & 1 & 6 & 37 & \\
\hline
\end{tabular}

